\title{
Ratio between carotid artery stiffness and blood flow - a new ultrasound index of ischemic leukoaraiosis
}

This article was published in the following Dove Press journal:

Clinical Interventions in Aging

25 January 2016

Number of times this article has been viewed

\author{
Monika Turk \\ Marjan Zaletel \\ Janja Pretnar-Oblak
}

Department of Vascular Neurology and Intensive Neurological Therapy, University Medical Centre Ljubljana, Ljubljana, Slovenia
Correspondence: Janja Pretnar-Oblak Department of Vascular Neurology and Intensive Neurological Therapy, University Medical Centre Ljubljana, Zaloska 2, 1000 Ljubljana, Slovenia Tel +386 4I5 | 466 I

$\mathrm{Fax}+3865222208$

Email janja.pretnar@kclj.si
Background: Ischemic leukoaraiosis (ILA) is associated with cognitive decline and aging. Its pathophysiology is believed to be ischemic in origin due to its association with cerebrovascular risk factors and similarity in location to lacunar infarctions. ILA diagnosis is still based on magnetic resonance imaging (MRI) as well as exclusion of other causes of white matter hyperintensities. So far, there are no known confirming diagnostic tests of ILA. Ultrasound studies have recently shown increased large artery stiffness, increased cerebrovascular resistance, and lower cerebral blood flow in patients with ILA. Increased arterial stiffness and decreased blood flow could have a synergistic effect, and their ratio could be a useful diagnostic index of ILA. Methods: In this post hoc analysis, we introduced new ILA indices (ILAi) that are ratios of the carotid stiffness parameters (pulse wave velocity beta [PWV $\beta$ ], pressure-strain elasticity modulus [Ep], $\beta$ index), and diastolic and mean blood flows in the internal carotid artery: Q-ICAd and Q-ICAm. We compared the ILAi of 52 patients with ILA and 44 sex- and risk factor-matched controls with normal MRI of the head. ILA diagnosis was based on MRI and exclusion of other causes of white matter hyperintensities. The diagnostic significance of ILAi for the prediction of ILA was analyzed.

Results: All ILAi significantly differed between the groups; the most significant were PWV $\beta$ / Q-ICAd (ILA group: $1.96 \pm 0.64$ vs control group: $1.56 \pm 0.40, P=0.001$ ) and PWV $\beta / Q-I C A m$ (ILA group: $1.13 \pm 0.32$ vs control group: $0.94 \pm 0.25, P=0.003)$. All ILAi were significantly associated with ILA $(P<0.01)$ and were significant independent predictors of ILA. All ILAi were also sensitive and specific for predicting ILA (area under the curve: $0.632-0.683, P<0.05$ ).

Conclusion: The new ultrasound indices significantly differed between patients with ILA and the control group and were significant predictors of ILA. A combination of lower carotid blood flow and increased carotid stiffness represented as ILAi probably has a diagnostic value in patients with ILA.

Keywords: carotid duplex ultrasound, carotid stiffness, carotid blood flow, white matter hyperintensities

\section{Introduction}

Leukoaraiosis is a neuroimaging term for hyperintensities in the periventricular and subcortical white matter, thus also called white matter hyperintensities (WMH). ${ }^{1}$ In its progressed form, leukoaraiosis is associated with cognitive decline, functional loss, psychiatric disorders, and gait disturbance. ${ }^{1}$ Its prevalence increases with age and reaches almost $100 \%$ in the eighth decade. ${ }^{2}$ Mostly due to the association with age and cerebrovascular disease (CVD) risk factors as well as similar location as lacunar infarctions, leukoaraiosis is believed to be ischemic in origin. ${ }^{3,4}$ Since the pathophysiological mechanisms of ischemic leukoaraiosis (ILA) are poorly understood, its 
diagnosis is sometimes made by extensive exclusion of other radiologically similar causes of $\mathrm{WMH}$ that require blood tests (hypovitaminosis, thyroid disease, rheumatic disease, Fabry's disease) and invasive lumbar puncture (intracranial vasculitis, demyelination, etc). ${ }^{1,3}$ So far, there are no known confirming diagnostic tests of ILA. Because of its mortality and morbidity, early diagnosis of ILA and its prompt treatment are very important. ${ }^{1,5}$

Ultrasound could be used as a noninvasive and easily applicable diagnostic test of ILA. It has been shown that aortic stiffness correlates with the development, progression, and degree of ILA. ${ }^{6-13}$ By one of the theories, increased large artery stiffness causes ILA by impaired damping of the arterial wave form and reduced wave reflection at the interface of large and small arteries that expose the small vessels in the brain to abnormal pressure. Increased flow pulsations could be the cause of damage to the small vessel wall. ${ }^{4,6,7,13}$ Furthermore, it has been shown that pulsatility of the middle cerebral artery and internal carotid artery (ICA) is higher, and the blood flow velocities are lower in patients with ILA independently of carotid atherosclerosis. ${ }^{6,7,14-19}$ Therefore, ILA could also be a consequence of the increased resistance of small arteries and hypoperfusion. ${ }^{14,15,18-21}$ Either of the theories is hard to prove.

In our previously published studies, we found increased carotid stiffness and diminished flow through the ICAs in patients with ILA compared to CVD risk factor-matched controls. $^{22,23}$ The combination of stiff large arteries with loss of the Windkessel effect and lower cerebral blood flow (CBF) could synergically cause insufficient diastolic perfusion in the deep white matter region and cause ILA. In this case, the ratio between local carotid stiffness parameters and CBF could be used as a diagnostic index of ILA. According to our knowledge, so far there are no studies on combined ultrasound markers of ILA.

The aim of this post hoc study was to compare the ratios between carotid stiffness parameters and carotid blood flow in patients with ILA to sex- and risk factor-matched control group in order to find a possible new ultrasound index of ILA.

\section{Methods}

Patients with ILA (ILA group) and age-, sex-, and CVD risk factor-matched controls without ILA (control group) participated in the study. The study was approved by the National Medical Ethics Committee of the Republic of Slovenia.

The study was a post hoc analysis of our previous studies on carotid stiffness parameters and carotid hemodynamic in patients with ILA. ${ }^{22,23}$ The subjects were included in the study based on the already performed brain magnetic resonance imaging (MRI). The patients with ILA were chosen among patients who have been treated in our outpatient clinic because of ILA and were up to 65 years old. Patients with acute stroke and patients with nonlacunar strokes or any intracranial pathology other than ILA were excluded based on the MRI findings and clinical data. ILA diagnosis was based on the WMH findings on brain MRI and some additional tests excluding other causes of WMH (demyelination, vasculitis, Fabry's disease). We screened all for hypovitaminosis (vitamin B12 and folic acid), thyroid hormones, vasculitis, chronic vessel inflammation factors (C-reactive proteins, homocysteine), and rheumatoid factors. Some had additional lumbar puncture, and besides basic tests, their cerebrospinal fluid was checked for demyelinization and the presence of oligoclonal bands.

All subjects were thoroughly examined, neurological status was assessed, blood pressure was measured, body mass index was calculated, alcohol, drugs, and cigarette consumption were noted, and 12-channel electrocardiogram and laboratory blood tests (hemogram, electrolytes, four-fractioned lipidogram) were performed to define CVD risk factors and additionally exclude nonvascular causes of WMH. We also excluded patients with cardiac arrhythmia, signs of ischemic heart disease, insulin-dependent diabetes, clinical signs of stroke, and $>50 \%$ carotid artery stenosis or hemodynamically important vertebral artery stenosis.

The control group subjects were selected among patients with similar CVD risk factors and already performed brain MRI that have been treated in neurological outpatient clinics because of other symptoms such as headache and vertigo. They were chosen based on the normal brain MRI and the exclusion criteria mentioned earlier. They were thoroughly examinedthe same protocol was followed as for the ILA group.

CVD risk factors were evaluated based on a standardized interview, which included patient history, clinical examination, body mass index determination, laboratory tests, blood pressure measurement, and electrocardiography. The laboratory tests included total cholesterol, low-density lipoprotein, high-density lipoprotein, and glucose.

\section{Experimental protocol}

The subjects were included in the study based on an already performed brain MRI. A brain MRI was performed in all patients using axial T1-weighted, T2-weighted, fluidattenuated inversion recovery, and proton-density-weighted scans on 1.5-T MRI scanner (Signal General Electric; GEMS) in the axial and sagittal planes with the slice thickness of 
$5 \mathrm{~mm}$. All MRI scans were evaluated by two experienced radiologists who were blinded to the ultrasound parameters, laboratory findings, and clinical variables of the study population.

All subjects gave informed consent to their participation in the study. The trial was performed in a quiet room under constant conditions. In the test preparation, each subject rested for 10-15 minutes. The mean arterial blood pressure and heart rate were measured continuously over a 5-minute interval using noninvasive plethysmography (Colin, CBM 7000).

Color-coded duplex sonography and power Doppler sonography equipped with echotracking system (Alpha 10, Aloka, Tokyo, Japan) of the carotid arteries were performed in all patients. Patients with unstable plaques of the carotid arteries and/or significant carotid artery stenosis ( $>50 \%)$ according to the ultrasonographic criteria were excluded from the study. Patients with significant asymmetry of the carotid flow were further investigated and in case of intracranial pathology excluded from the study. Patients with hemodynamically important stenosis or occlusion of the extracranial vertebral arteries were also excluded.

All measurements were obtained after $\geq 5$ minutes of rest in supine position in a quiet room with constant room temperature. We measured the blood flow velocities and diameter of both common carotid arteries (CCAs) $2 \mathrm{~cm}$ below the bulbus and blood flow velocity and mean diameter in both ICAs at the same place at the most clearly visible point distally from the bulbus. Average systolic, diastolic, and mean blood flow velocities, pulsatility index (PI), and resistivity index $(\mathrm{RI})$ were calculated using the average values of the left and right ICA in Equations (EQs) 1-3 (ICA systolic velocity and ICA end diastolic velocity). Diastolic and systolic blood flow was calculated using EQ 4, whereas we used peek systolic and end diastolic blood flow velocities and mean diameter of ICAs. Since we used the mean diameter of ICA, the blood flows are only the approximation of the real systolic and diastolic blood flows.

EQ 1. Mean velocity: (v-ICAs - v-ICAd)/3 + v-ICAd; v-ICAs: systolic velocity in ICA, v-ICAd: diastolic velocity in ICA

EQ 2. PI: (v-ICAs - v-ICAd)/mean velocity

EQ 3. RI: (v-ICAs - v-ICAd)/v-ICAs

EQ 4. Blood flow: $Q=v \pi(D / 2)^{2} ; v=$ velocity, $D=$ diameter of ICA.

Intima-media thickness (IMT), carotid diameter measurements, and arterial stiffness measurements were performed in the longitudinal way, strictly perpendicular to the ultrasound beam, with both walls clearly visualized bilaterally $2 \mathrm{~cm}$ below the bifurcation, on the far wall of the CCA. A highquality image was acquired along a minimum of $1.5 \mathrm{~cm}$ length of CCA segment for reproducible measurements. IMT was measured in accordance with the Mannheim. The distance between the characteristic echoes from the lumenintima and media-adventitia interfaces was measured. The final IMT value was based on the mean value of the three maximal IMT measurements in three frames. Arterial stiffness was automatically measured from the modification of the arterial diameter between the systolic and diastolic phases on CCA segments. Carotid diameter waveforms were assessed by means of an ultrasound and converted to carotid pressure waveforms using an empirically derived exponential relationship between pressure and arterial crosssection. Blood pressure measurements were obtained simultaneously with ultrasound measurements. The derived carotid pressure waveform was calibrated from brachial end diastolic and mean arterial pressures by iteratively changing the wall rigidity coefficient. ${ }^{24}$ This allowed the calculation of the arterial stiffness indices obtained as the mean value of the last six measurements:

- $\quad \beta$, beta stiffness: $\beta=\ln (P s / P d) /([D s-D d] / D d) ; P s$ and $P d$ are the systolic and diastolic pressure in $\mathrm{mmHg}, D s$ and $D d$ are the systolic and diastolic carotid diameters, and $\ln$ is the natural logarithm. ${ }^{25}$

- Ep $(\mathrm{kPa})$, epsilon, pressure-strain elasticity modulus: $\mathrm{Ep}=(P s-P d) /([D s-D d] / D d) \cdot{ }^{25,26}$

- $\operatorname{PWV} \beta(\mathrm{m} / \mathrm{s})$, one-point pulse wave velocity: PWV $\beta$ : $\sqrt{([\beta \times \mathrm{Pd}] / 2 \rho)} ; \rho$ is the blood density, $\rho=1,050 \mathrm{~kg} / \mathrm{m}^{3} .^{27}$

We then introduced new ILA indices (ILAi) that are the ratios of local carotid stiffness parameters (pulse wave velocity beta [PWV $\beta$ ], pressure-strain elasticity modulus [Ep], $\beta$ index) and mean and diastolic blood flows in ICA

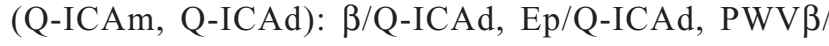
Q-ICAd, $\beta / \mathrm{Q}-\mathrm{ICAm}$, Ep/Q-ICAm, and PWV $\beta / \mathrm{Q}-\mathrm{ICAm}$.

\section{Statistical analyses}

All statistical analyses were performed using SPSS 20.0 software (IBM Corporation, Armonk, NY): subject characteristics and the following ultrasound variables such as PWV $\beta$, Ep, $\beta$ index, Q-ICAd, systolic blood flow in ICA (Q-ICAs), and Q-ICAm were compared between the ILA and control group by independently paired $t$-test. The ILAi were calculated and compared between the two groups using paired $t$-test. Standardization of all the variables was made by calculation of $Z$-scores for all the ultrasound variables, and the $Z$-scores were used in all further analyses. 
Correlation calculations between ultrasound parameters and ILA were performed by Spearman's correlation coefficient. Linear regression and logistic multivariate regression were used to analyze the associations between US parameters. Furthermore, logistic regression tests were used to evaluate which ultrasound parameters could predict ILA. For the evaluation of the predictive value of ultrasound parameters to identify patients with ILA, the area under the receiver operating characteristic (ROC) curve (AUC) and the corresponding $95 \%$ confidence interval (CI) were calculated. A $P$-value of $<0.05$ was considered as statistically significant.

\section{Results}

Fifty-two patients, aged 54.90 \pm 8.27 years, with ILA (ILA group) and 44 age-, sex-, and CVD risk factor-matched controls without ILA (control group) were included in the study. The clinical characteristics including baseline demographic data and risk factors for CVD of the ILA group and the control group are presented in Table 1.

The parameters of the ILA patients that significantly differed from the control group in our previous studies were used for the calculation of ILAi. ILAi were therefore defined as the ratios between carotid stiffness parameters (PWV $\beta$, Ep, $\beta$ index) and blood flows in the ICA: Q-ICAd and Q-ICAm. ${ }^{22,23}$ All the ILAi were higher in the ILA group compared to the

Table I Clinical and laboratory data of the patients with ILA and controls

\begin{tabular}{llll}
\hline Variable & ILA group & Control group & $P$-value \\
\hline Patients (n) & 52 & 44 & \\
Age (years) & $54.90 \pm 8.27$ & $52.39 \pm 7.34$ & 0.118 \\
Male:female & $1.27 \pm 0.45$ & $1.32 \pm 0.47$ & 0.605 \\
BMI $\left(\mathrm{kg} / \mathrm{m}^{2}\right)$ & $30.40 \pm 4.73$ & $30.10 \pm 5.5 \mathrm{I}$ & 0.778 \\
Arterial hypertension (\%) & $34(0.65)$ & $24(0.55)$ & 0.286 \\
MAP (mmHg) & $103.30 \pm 18.77$ & $105.06 \pm 14.49$ & 0.617 \\
HR (beats/min) & $61.09 \pm \mathrm{II} .00$ & $62.92 \pm 9.98$ & 0.415 \\
Glucose (mmol/L) & $5.3 \mathrm{I} \pm 0.75$ & $5.20 \pm 0.87$ & 0.530 \\
Smoking (\%) & $9(0.17)$ & $8(0.18)$ & 0.912 \\
Hypercholesterolemia & $34(0.65)$ & $30(0.68)$ & 0.775 \\
Cholesterol (mmol/L) & $5.03 \pm \mathrm{I} .18$ & $5.28 \pm \mathrm{I} .12$ & 0.299 \\
LDL (mmol/L) & $3.1 \mathrm{I} \pm \mathrm{I} .0 \mathrm{I}$ & $3.27 \pm 0.99$ & $0.44 \mathrm{I}$ \\
HDL (mmol/L) & $1.29 \pm 0.39$ & $1.23 \pm 0.29$ & 0.366 \\
Triglycerides (mmol/L) & $\mathrm{I} .76 \pm \mathrm{I} . \mathrm{I} 4$ & $\mathrm{I} .89 \pm \mathrm{I} .2 \mathrm{I}$ & 0.608 \\
Antiaggregation & $20(0.38)$ & $7(0.16)$ & 0.012 \\
therapy (\%) & & & \\
Antihypertensive & $28(0.54)$ & $22(0.50)$ & $0.7 \mathrm{II}$ \\
therapy (\%) & & & \\
Statin therapy (\%) & $\mathrm{I}(0.29)$ & $\mathrm{II}(0.25)$ & 0.675 \\
\hline
\end{tabular}

Notes: Data are presented as mean \pm standard deviation for continuous variables and number of subjects (percentage) for categorical variables and $P$-value.

Abbreviations: ILA, ischemic leukoaraiosis; BMI, body mass index; LDL, low-density lipoprotein; HDL, high-density lipoprotein; HR, heart rate; MAP, mean arterial pressure.
Table 2 Ultrasound parameters of carotid arterial stiffness, carotid blood flow, and ratios of carotid stiffness and blood flow in the ILA group and the control group

\begin{tabular}{|c|c|c|c|}
\hline Variable & ILA group & Control group & $P$-value \\
\hline$\beta$ & $7.57 \pm 2.79$ & $6.70 \pm I .77$ & $0.07 I$ \\
\hline Ep $(\mathrm{kPa})$ & || $5.9 \mid \pm 49.07$ & $97.97 \pm 29.73$ & 0.038 \\
\hline$P W V \beta(m / s)$ & $6.47 \pm 1.23$ & $5.94 \pm 0.87$ & 0.017 \\
\hline Q-ICAs $\left(\mathrm{mm}^{3} / \mathrm{s}\right)$ & $10.962 \pm 2.18$ & $11.96 \pm 2.87$ & 0.070 \\
\hline Q-ICAd $\left(\mathrm{mm}^{3} / \mathrm{s}\right)$ & $3.50 \pm 0.88$ & $3.98 \pm 0.80$ & 0.008 \\
\hline Q-ICAm $\left(\mathrm{mm}^{3} / \mathrm{s}\right)$ & $5.99 \pm 1.23$ & $6.64 \pm 1.44$ & 0.023 \\
\hline$\beta / Q-I C A m\left(s / m^{3}\right)$ & $1.31 \pm 0.55$ & $1.04 \pm 0.36$ & 0.008 \\
\hline Ep/Q-ICAm (kPa s/mm³) & $20.20 \pm 9.66$ & $15.50 \pm 5.89$ & 0.006 \\
\hline PWV $\beta / Q-I C A m\left(\mathrm{~mm}^{-2}\right)$ & $1.13 \pm 0.32$ & $0.94 \pm 0.25$ & 0.003 \\
\hline$\beta / \mathrm{Q}-I C A d\left(\mathrm{~s} / \mathrm{mm}^{3}\right)$ & $2.27 \pm 1.07$ & $1.73 \pm 0.60$ & 0.003 \\
\hline Ep/Q-ICAd (kPa s/mm³) & $35.04 \pm 18.59$ & $25.73 \pm 9.65$ & 0.003 \\
\hline PWV $\beta / Q-I C A d\left(m^{-2}\right)$ & $1.96 \pm 0.64$ & $1.56 \pm 0.40$ & 0.001 \\
\hline
\end{tabular}

Notes: Data are presented as mean \pm standard deviation, $P$-value, and $95 \%$ confidence interval of the difference.

Abbreviations: ILA, ischemic leukoaraiosis; Ep, pressure-strain elasticity modulus; PWV $\beta$, pulse wave velocity beta; $\beta$, beta index; Q-ICAs, average systolic blood flow in internal carotid arteries; Q-ICAd, average diastolic blood flow in internal carotid arteries; Q-ICAm, average mean blood flow in internal carotid arteries.

control group $(P<0.01)$, the most significant was $P W V \beta /$ Q-ICAd $(P=0.001)$. The results are shown in Table 2 .

The correlations of all the ultrasound parameters with ILA are shown in Table 3. PWV $\beta$, Q-ICAd, Q-ICAm, and all the ILAi significantly correlated with ILA (Spearman's correlation coefficient: $r 0.227-0.315, P<0.03$ for all ILAi). The results were the same when using standardized $Z$-scores of variables.

Linear regression analysis adjusted for sex, age, and all other CVD risk factors mentioned in Table 1 showed no associations between stiffness parameters and systolic, diastolic, and mean carotid blood flow. There were also no associations

Table 3 Correlations of ultrasound parameters of ILA with ILA

\begin{tabular}{lll}
\hline Variable & Spearman's $\rho$ & P-value \\
\hline$\beta$ & 0.149 & 0.153 \\
Ep $(\mathrm{kPa})$ & 0.166 & 0.122 \\
$\mathrm{PWV} \beta(\mathrm{m} / \mathrm{s})$ & 0.233 & 0.030 \\
Q-ICAd $\left(\mathrm{mm}^{3} / \mathrm{s}\right)$ & -0.286 & 0.005 \\
Q-ICAm $\left(\mathrm{mm}^{3} / \mathrm{s}\right)$ & -0.232 & 0.025 \\
$\beta / Q-I C A m\left(\mathrm{~s} / \mathrm{mm}^{3}\right)$ & 0.227 & 0.031 \\
Ep/Q-ICAm $\left(\mathrm{kPa} \mathrm{s}^{2} / \mathrm{mm}^{3}\right)$ & 0.233 & 0.030 \\
PWV $\beta / Q-I C A m ~\left(\mathrm{~mm}^{-2}\right)$ & 0.254 & 0.018 \\
$\beta / Q-I C A d\left(\mathrm{~s} / \mathrm{mm}^{3}\right)$ & 0.252 & 0.016 \\
Ep/Q-ICAd $\left(\mathrm{kPa} \mathrm{s} / \mathrm{mm}^{3}\right)$ & 0.257 & 0.016 \\
PWV $\beta / Q-I C A d\left(\mathrm{~mm}^{-2}\right)$ & 0.315 & 0.003 \\
\hline
\end{tabular}

Notes: Data are presented as Spearman's rho and $P$-value. Z-scores of variables were used in the analyses.

Abbreviations: ILA, ischemic leukoaraiosis; Ep, pressure-strain elasticity modulus; PWV $\beta$, one-point pulse wave velocity beta; $\beta$, beta index; Q-ICAd, average diastolic blood flow in internal carotid arteries; Q-ICAm, average mean blood flow in internal carotid arteries. 
Table 4 Associations of ultrasound parameters of ILA with ILA

\begin{tabular}{|c|c|c|c|c|}
\hline \multirow[t]{2}{*}{ Variable } & \multirow[t]{2}{*}{ OR } & \multirow[t]{2}{*}{$P$-value } & \multicolumn{2}{|l|}{$\mathrm{Cl}$} \\
\hline & & & Lower & Upper \\
\hline$\beta$ & 1.472 & 0.090 & $0.94 I$ & 2.303 \\
\hline Ep (kPa) & 1.607 & 0.055 & 0.990 & 2.610 \\
\hline$P W V \beta(m / s)$ & 1.712 & 0.028 & 1.061 & 2.762 \\
\hline Q-ICAd $\left(\mathrm{mm}^{3} / \mathrm{s}\right)$ & $0.54 I$ & 0.008 & 0.343 & 0.853 \\
\hline Q-ICAm $\left(\mathrm{mm}^{3} / \mathrm{s}\right)$ & 0.577 & 0.016 & 0.368 & 0.902 \\
\hline$\beta / Q-I C A m\left(s / m^{3}\right)$ & 1.848 & 0.016 & 1.120 & 3.050 \\
\hline Ep/Q-ICAm (kPa s/mm³) & 1.946 & 0.015 & 1.140 & 3.323 \\
\hline$P W V \beta / Q-I C A m\left(m^{-2}\right)$ & 2.009 & 0.007 & 1.207 & 3.343 \\
\hline$\beta / \mathrm{Q}-\mathrm{ICAd}\left(\mathrm{s} / \mathrm{mm}^{3}\right)$ & 2.152 & 0.010 & 1.204 & 3.847 \\
\hline Ep/Q-ICAd (kPa s/mm³) & 2.249 & 0.010 & 1.214 & 4.167 \\
\hline PWV $\beta / Q-I C A d\left(\mathrm{~mm}^{-2}\right)$ & 2.441 & 0.003 & 1.350 & 4.412 \\
\hline
\end{tabular}

Notes: Logistic regression: ILA group as a dependent variable, standardized $Z$-scores of variables were used in the analyses. Data are presented as OR, $P$-value, and $95 \% \mathrm{Cl}$ of OR.

Abbreviations: ILA, ischemic leukoaraiosis; OR, odds ratio; $\mathrm{Cl}$, confidence interval of OR; Ep, pressure-strain elasticity modulus; $P W V \beta$, one-point pulse wave velocity beta; $\beta$, beta index; Q-ICAd, average diastolic blood flow in internal carotid arteries; Q-ICAm, average mean blood flow in internal carotid artery.

between mean carotid blood flow and PI and RI, whereas systolic and diastolic blood flows were independently significantly associated with PI and RI: Q-ICAs with PI-ICA: standardized $B=0.231, P=0.026$, $C I=0.028,0.434$; Q-ICAs with RI-ICA: standardized $B=0.233, P=0.024, C I=0.031,0.436$; Q-ICAd with PI-ICA: standardized $B=-0.402, P<0.001$, $\mathrm{CI}=-0.593,-0.211$; and Q-ICAd with RI: standardized $B=-0.399, P<0.001, C I=-0.590,-0.208$.

The multivariate logistic regression model showed that PWV $\beta / Q-I C A d$ and PWV $\beta / Q-I C A m$ were the strongest independent predictors of ILA with odds ratio $(\mathrm{OR})=9.636$ for $\mathrm{PWV} \beta / \mathrm{Q}-\mathrm{ICAm}(P=0.007,95 \% \mathrm{CI}=1.841,50.443)$ and 4.61 for $\mathrm{PWV} \beta / \mathrm{Q}-\mathrm{ICAd}(P=0.003,95 \% \mathrm{CI}=1.674,12.743)$. When using standardized $Z$-scores in the analyses, the strongest was still $\mathrm{PWV} \beta / \mathrm{Q}-\mathrm{ICAd}$ (OR $=2.441, P=0.003,95 \%$ $\mathrm{CI}=1.350,4.412)$. The other results are shown in Table 4.

For the evaluation of the diagnostic significance of ILAi for the prediction of ILA, the AUC was computed (Table 5). The highest AUC was calculated for PWV $\beta /$ Q-ICAd (AUC $=0.683,95 \% \mathrm{CI}=0.572,0.795, P=0.004$ ). The results were the same when using standardized $Z$-scores in the analyses.

\section{Discussion}

The main finding of this study was that the ratios between carotid stiffness parameters and carotid blood flows were significantly higher in the ILA group compared to the risk factor-matched control group without ILA. These ratios represented as new ILAi were significant predictors of ILA. The new ultrasound indices offer a better insight into the
Table 5 ROC analyses of ultrasound parameters of ILA

\begin{tabular}{lllll}
\hline Variable & AUC & P-value & \multicolumn{2}{l}{$\mathbf{9 5 \%} \mathbf{C l}$} \\
\cline { 4 - 5 } & & & Lower & Upper \\
\hline$\beta$ & 0.587 & 0.152 & 0.470 & 0.704 \\
Ep $(\mathrm{kPa})$ & 0.597 & 0.121 & 0.478 & 0.715 \\
PWV $(\mathrm{m} / \mathrm{s})$ & 0.636 & 0.031 & 0.518 & 0.753 \\
Q-ICAm $\left(\mathrm{mm}^{3} / \mathrm{s}\right)$ & 0.365 & 0.026 & 0.250 & 0.480 \\
Q-ICAd $\left(\mathrm{mm}^{3} / \mathrm{s}\right)$ & 0.333 & 0.006 & 0.224 & 0.443 \\
$\beta / Q-I C A m\left(\mathrm{~s} / \mathrm{mm}^{3}\right)$ & 0.632 & 0.032 & 0.518 & 0.747 \\
Ep/Q-ICAm $\left(\mathrm{kPa} \mathrm{s} / \mathrm{mm}^{3}\right)$ & 0.635 & 0.031 & 0.519 & 0.752 \\
PWV $\beta / Q-I C A m\left(\mathrm{~mm}^{-2}\right)$ & 0.648 & 0.019 & 0.532 & 0.764 \\
$\beta / Q-I C A d\left(\mathrm{~s} / \mathrm{mm}^{3}\right)$ & 0.647 & 0.017 & 0.534 & 0.760 \\
Ep/Q-ICAd $\left(\mathrm{kPa} \mathrm{s} / \mathrm{mm}^{3}\right)$ & 0.650 & 0.017 & 0.535 & 0.764 \\
PWV $\beta / Q-I C A d\left(\mathrm{~mm}^{-2}\right)$ & 0.683 & 0.004 & 0.572 & 0.795 \\
\hline
\end{tabular}

Notes: Data are presented as area under ROC curve, $P$-value, and $95 \% \mathrm{Cl}$. $Z$-scores of variables were used in the analyses.

Abbreviations: ILA, ischemic leukoaraiosis; AUC, area under ROC curve; ROC, receiver operating characteristic; $\mathrm{Cl}$, confidence interval; Ep, pressure-strain elasticity modulus; PWV $\beta$, one-point pulse wave velocity beta; $\beta$, beta index; Q-ICAd, average diastolic blood flow in internal carotid arteries; Q-ICAm, average mean blood flow in internal carotid arteries.

pathophysiology of ILA. ILAi could be used as a screening test for ILA for patients with high CVD risk requiring further more expensive diagnosis including MRI. Besides, ILAi may be useful in the diagnostics of ILA possibly predicting the so-called "ischemic" etiology of WMH seen on MRI.

Our study was the first one to combine local carotid stiffness parameters and ICA blood flow in order to find useful ILAi. It is also the first study that compared ICA blood flow parameters and local carotid stiffness parameters of ILA patients to the age-, sex- and CVD risk factor-matched control group without ILA. This study is a post hoc analysis of our previous two studies. ${ }^{22,23}$ In the first study, we studied carotid stiffness parameters in patients with ILA, which had not been done previously, ${ }^{22}$ although they are some of the most commonly used markers of local arterial stiffness. ${ }^{25-28}$ In the second study, blood flow velocities and flows through the carotid arteries in patients with ILA were studied. Lower flows were found, thus suggesting hypoperfusion mechanism of ILA. ${ }^{23}$ In both studies, we found useful carotid ultrasound indices of ILA. In this study, we combined ultrasound parameters of carotid stiffness and blood flow. Regarding general knowledge about direct influence of arterial stiffness on systolic and diastolic blood flow velocity and other hemodynamic adaptations to this, the mean blood flow should be almost independent of stiffness. ${ }^{21}$ However, it is not possible to exclude indirect associations between the two. For this purpose, we tested the associations between the stiffness parameters and carotid blood flow after adjustment for CVD risk factors. The linear regression and multivariate logistic regression model showed that the stiffness parameters and 
ICA blood flows were independently associated with ILA. Thus, we used them in the ratios as the new ILAi. All the ILAi were significantly higher in the ILA group $(P<0.01)$ and significantly correlated with ILA $(P<0.05)$. The logistic regression model showed that they are better independent predictors of ILA than carotid stiffness and blood flow parameters alone. Similarly, the diagnostic significance of ILAi tested by the ROC curves showed that ILAi are more sensitive and specific for predicting ILA (AUC $>0.600, P<0.05$ ) than carotid stiffness and blood flow parameters themselves. Therefore, the ratio between local carotid stiffness parameters and $\mathrm{CBF}$ could be a useful diagnostic index of ILA. Among all the ILAi, the strongest index was PWV $\beta / Q-I C A d$, but according to the well-known influence of arterial stiffness on diastolic blood flow, ${ }^{29}$ the index PWV $\beta / Q-I C A m$ should be clinically more reliable. However, we have to stress out that although significant, the AUCs for all ILAi were relatively low, and the specificity and sensitivity were not high enough for diagnosing ILA by themselves. However, ILAi may be useful in predicting the ischemic etiology of leukoaraiosis seen on head MRI avoiding other more expensive and invasive diagnostic tests. Even more importantly, ILAi could possibly be used as a screening test for ILA in subjects at risk even before MRI is performed.

A major advantage of this study was the usage of the commonly available noninvasive and easily applicable ultrasound methodology. We used color-coded duplex sonography and power Doppler sonography equipped with a high-resolution B-mode echo-tracking system that enables measurements of ICA hemodynamic parameters and direct and easy determination of the local arterial stiffness parameters. ${ }^{26-29}$ The advantages and usage of these methods were explained in our previous article..$^{22,23}$

In our previous article, ${ }^{23}$ we assumed that diastolic hypoperfusion was the leading mechanism of ILA. However, the PI, which was also higher in our study, ${ }^{23}$ and increased carotid stiffness parameters in ILA patients could both suggest another theory by which the mechanism of ILA could be a microvascular injury associated with the transmission of excessive flow pulsatility into the cerebral microcirculation due to an increased central artery stiffness and decreased wave reflection at the interface of large and small arteries. ${ }^{4,6,7,13}$ Since different theories about hemodynamic pathological mechanisms of ILA are probably not mutually exclusive, neither lower blood flow in the ICA nor the increased carotid arterial stiffness is probably enough for the development of ILA. According to our results, we assumed that the presence of the stiff large arteries in combination with the increased resistance of small arteries that lowers CBF could act synergistically and cause ILA. Since patients with ILA have higher vascular resistance, ${ }^{6,7,14-18}$ higher mean arterial pressure is needed to obtain the same CBF. ${ }^{21}$ On the other hand, stiff large arteries impair the damping of the arterial waveform, and this might cause insufficient cerebral perfusion throughout diastole at the lower levels of mean arterial pressure. ${ }^{20}$ The significantly lower diastolic flows in patients with ILA support this theory. Therefore, the ratios between the carotid stiffness parameters and diastolic blood flow could be useful indices of ILA.

Although the underlying mechanism is not well understood, our results could be used to optimize the prevention strategies for ILA. Even though hypertension is one of the main risk factors of ILA, the patients at risk should not be treated too rigorously in order not to cause additional cerebral hypoperfusion. ${ }^{30}$

\section{Conclusion}

In conclusion, the new ILAi defined as the ratios between the carotid stiffness parameters and blood flow are significant predictors of ILA and have a potential diagnostic value in patients with ILA. They could be implemented as a screening tool to evaluate the risk of ILA before MRI is done. ILAi could potentially also be used in distinguishing ischemic from nonischemic causes of WMH seen on MRI. Larger studies would be necessary in order to confirm that.

\section{Acknowledgments}

We would like to thank the medical technicians and nurses of the Department of Vascular Neurology and Intensive Neurological Therapy, University Medical Centre Ljubljana, for their kind help in the clinical examinations and measurements. Special thanks goes to neurologist Matija Zupan, MD, medical technician Tine Beznik, and the neuroradiologist Katarina Šurlan Popović, MD, PhD. The study received no grants or external funding.

\section{Disclosure}

The authors report no conflicts of interest in this work.

\section{References}

1. The LADIS study group. A decade of the LADIS (leukoaraiosis and disability) study: what have we learned about white matter changes and small-vessel disease? Cerebrovasc Dis. 2011;32:577-588.

2. De Leeuw FE, de Groot JC, Achten E, et al. Prevalence of cerebral white matter lesions in elderly people: a population based magnetic resonance imaging study. The Rotterdam Scan Study. Neurol Neurosurg Psychiatry. 2001;70:9-14.

3. Pantoni L. Cerebral small vessel disease: from pathogenesis and clinical characteristics to therapeutic challenges. Lancet Neurol. 2010;9: 689-701. 
4. Poels MM, Zaccai K, Verwoert GC, et al. Arterial stiffness and cerebral small vessel disease: the Rotterdam Scan Study. Stroke. 2012;43: 2637-2642.

5. Bokura H, Kobayashi S, Yamaguchi S, et al. Silent brain infarction and subcortical white matter lesions increase the risk of stroke and mortality: a prospective cohort study. J Stroke Cerebrovasc Dis. 2006;15: 57-63.

6. Mitchell GF, van Buchem MA, Sigurdsson S, et al. Arterial stiffness, pressure and flow pulsatility and brain structure and function: the Age, Gene/Environment Susceptibility-Reykjavik Study. Brain. 2011; 134:3398-3407.

7. Webb AJ, Simoni M, Mazzucco S, et al. Increased cerebral arterial pulsatility in patients with leukoaraiosis: arterial stiffness enhances transmission of aortic pulsatility. Stroke. 2012;43:2631-2636.

8. Brisset M, Boutouyrie P, Pico F, et al. Large-vessel correlates of cerebral small-vessel disease. Neurology. 2013;80:662-669.

9. Kearney-Schwartz A, Rossignol P, Bracard S, et al. Vascular structure and function is correlated to cognitive performance and white matter hyperintensities in older hypertensive patients with subjective memory complaints. Stroke. 2009;40:1229-1236.

10. van Elderen SG, Brandts A, Westenberg JJ, et al. Aortic stiffness is associated with cardiac function and cerebral small vessel disease in patients with type 1 diabetes mellitus: assessment by magnetic resonance imaging. Eur Radiol. 2010;20:1132-1138.

11. Henskens LH, Kroon AA, van Oostenbrugge RJ, et al. Increased aortic pulse wave velocity is associated with silent cerebral small-vessel disease in hypertensive patients. Hypertension. 2008;52:1120-1126.

12. Hatanaka R, Obara T, Watabe D, et al. Association of arterial stiffness with silent cerebrovascular lesions: the Ohasama study. Cerebrovasc Dis. 2011;31:329-337.

13. O'Rourke MF, Safar ME. Relationship between aortic stiffening and microvascular disease in brain and kidney: cause and logic of therapy. Hypertension. 2005;46:200-204.

14. Tanaka T, Shimizu T, Fukuhara T. The relationship between leukoaraiosis volume and parameters of carotid artery duplex ultrasonographic scanning in asymptomatic diabetic patients. Comput Med Imaging Graph. 2009;33:489-493

15. Aribisala BS, Morris Z, Eadie E, et al. Blood pressure, internal carotid artery flow parameters, and age-related white matter hyperintensities. Hypertension. 2014;63:1011-1018.

16. Heliopoulos I, Artemis D, Vadikolias K, et al. Association of ultrasonographic parameters with subclinical white-matter hyperintensities in hypertensive patients. Cardiovasc Psychiatry Neurol. 2012;2012: 616572 .
17. Sierra C, de la Sierra A, Chamorro A, et al. Cerebral hemodynamics and silent cerebral white matter lesions in middle-aged essential hypertensive patients. Blood Press. 2004;13:304-309.

18. Fukuhara T, Hida K, Manabe Y, et al. Reduced flow velocity in the internal carotid artery independently of cardiac hemodynamics in patients with cerebral ischemia. J Clin Ultrasound. 2007;35:314-321.

19. Henry-Feugeas MC, Onen F, Claeys ES. Classifying late-onset dementia with MRI: is arteriosclerotic brain degeneration the most common cause of Alzheimer's syndrome? Clin Interv Aging. 2008;3:187-199.

20. O’Sullivan M, Lythgoe DJ, Pereira AC, et al. Patterns of cerebral blood flow reduction in patients with ischemic leukoaraiosis. Neurology. 2002; 59:321-326.

21. Mitchell GF. Effects of central arterial aging on the structure and function of the peripheral vasculature: implications for end-organ damage. J Appl Physiol. 2008;105:1652-1660.

22. Turk M, Pretnar-Oblak J, Zupan M, et al. Ultrasound diagnostics of carotid arterial stiffness in patients with ischemic leukoaraiosis. Ultrasound Med Biol. 2015;41:64-71.

23. Turk M, Zupan M, Zaletel M, et al. Carotid arterial hemodynamic in ischemic levkoaraiosis suggest hypoperfusion mechanism. Eur Neurol. 2015;73:310-315.

24. Meinders JM, Hoeks APG. Simultaneous assessment of diameter and pressure waveforms in the carotid artery. Ultrasound Med Biol. 2004;30:147-154.

25. Rhee MY, Lee HY, Park YB. Measurements of arterial stiffness: methodological aspects. Korean Circ J. 2008;38:343-350.

26. Laurent S, Cockcroft J, Van Bortel L, et al. Expert consensus document on arterial stiffness: methodological issues and clinical applications. Eur Heart J. 2006;27:2588-2605.

27. Jaroch J, Loboz Grudzień K, Bociąga Z, et al. The relationship of carotid arterial stiffness to left ventricular diastolic dysfunction in untreated hypertension. Kardiol Pol. 2012;70:223-231.

28. Kawasaki T, Sasayaa S, Yagi S, et al. Non-invasive assessment of the age related changes in stiffness of major branches of the human arteries. Cardiovasc Res. 1987;21:678-687.

29. Albayrak R, Degirmenci B, Acar M, et al. Doppler sonography evaluation of flow velocity and volume of the extracranial internal carotid and vertebral arteries in healthy adults. J Clin Ultrasound. 2007;35: 27-33.

30. Denker MG, Cohen DL. What is an appropriate blood pressure goal for the elderly: review of recent studies and practical recommendations. Clin Interv Aging. 2013;8:1505-1517. Epub 2013 Nov 14.
Clinical Interventions in Aging

\section{Publish your work in this journal}

Clinical Interventions in Aging is an international, peer-reviewed journal focusing on evidence-based reports on the value or lack thereof of treatments intended to prevent or delay the onset of maladaptive correlates of aging in human beings. This journal is indexed on PubMed Central, MedLine,

\section{Dovepress}

CAS, Scopus and the Elsevier Bibliographic databases. The manuscript management system is completely online and includes a very quick and fair peer-review system, which is all easy to use. Visit http://www.dovepress. com/testimonials.php to read real quotes from published authors. 